\title{
HEART
}

\section{Stress echocardiography for selecting potential responders to cardiac resynchronisation therapy}

Marie Moonen, Kim O'Connor, Julien Magne, et al.

Heart 2010 96: 1142-1146

doi: $10.1136 /$ hrt.2010.199828

Updated information and services can be found at:

http://heart.bmj.com/content/96/14/1142.full.html

These include:

References This article cites 41 articles, 29 of which can be accessed free at: http://heart.bmj.com/content/96/14/1142.full.html\#ref-list-1

Email alerting Receive free email alerts when new articles cite this article. Sign up in the service box at the top right corner of the online article.

Notes

To order reprints of this article go to:

http://heart.bmj.com/cgi/reprintform

To subscribe to Heart go to:

http://heart.bmj.com/subscriptions 


\title{
Stress echocardiography for selecting potential responders to cardiac resynchronisation therapy
}

\author{
Marie Moonen, Kim O'Connor, Julien Magne, Patrizio Lancellotti, Luc A Pierard
}

Department of Cardiology, University Hospital Sart Tilman, Liège, Belgium

\section{Correspondence to}

Professor Luc A Pierard

Department of Cardiology, University Hospital Sart Tilman, Liège B-4000, Belgium; Ipierard@chu.ulg.ac.be

MM is a Research Fellow of the Belgian F.R.S.-FNRS

Accepted 27 April 2010

\begin{abstract}
In the current ESC/ACC/AHA guidelines, the selection of patients for cardiac resynchronisation therapy (CRT) is based upon the QRS duration, which reflects interventricular dyssynchrony. However, about 30\% of patients do not respond to CRT. It has previously been demonstrated that the presence of left ventricular mechanical dyssynchrony is predictive of response to CRT after implantation. Most criteria assessing such dyssynchrony were derived from data obtained with resting Doppler echocardiography. The recently published PROSPECT (Predictors of Response to CRT) trial failed to identify echocardiographic measures of dyssynchrony that could routinely be recommended for patient selection before CRT implantation. Therefore, solutions may come from other echocardiographic modalities, such as dobutamine stress echocardiography and exercise echocardiography. The purpose of this review is to evaluate the usefulness of stress echocardiography to predict response to CRT. This review will show how exercise-induced changes in dyssynchrony and severity of mitral regurgitation and the role of preserved contractile reserve may help to better identify potential responders.
\end{abstract}

Despite significant improvement in medical treatment, severe heart failure remains associated with markedly reduced quality of life, persistent symptoms and a high mortality rate. Cardiac resynchronisation therapy (CRT) has emerged as a potential adjunctive treatment in patients with drug-refractory heart failure. ${ }^{1}$ CRT, associated or not with defibrillator function, is currently recommended in European Society of Cardiology guidelines (class I, level of evidence A) to reduce morbidity and mortality in patients who remain symptomatic (New York Heart Association (NYHA) class III-IV) despite optimal stable medical treatment in association with reduced left ventricular (LV) ejection fraction (EF) (LVEF <35\%) and wide ORS (ORS width $>120 \mathrm{~ms}$ ) (box 1). ${ }^{2}$ CRT has been shown to improve symptoms, quality of life, exercise capacity, LV performance and survival in two major trials: COMPANION (Comparison of Medical Therapy, Pacing, and Defibrillation in Heart Failure) and CARE-HF (Cardiac Resynchronisation-Heart Failure). ${ }^{3} 4$

Historically, CRT has been conceptualised by electrophysiologists and is based on the observation that severe heart failure is frequently associated with interventricular conduction disordersnamely, wide ORS, which could be responsible for asynchronous LV contraction and decreased LV pumping function. Normally, an efficient LV contraction requires a global LV depolarisation around $40 \mathrm{~ms}$ variation in the onset of electrical activation throughout the wall and very similar lowlevel variability in the timing of mechanical activation. ${ }^{5}$ However, when ORS duration is used as a selection criterion for CRT, about $30 \%$ of nonresponders are encountered. ${ }^{6}$ The ORS width as a marker of electrical dyssynchrony may not reflect the extent of the mechanical dyssynchrony. As delayed myocardial electrical activation did not appear to be an adequate option, interest has been focused on delayed myocardial contraction-namely, cardiac mechanical dyssynchrony. Dyssynchrony has three components: atrioventricular, interventricular and intraventricular. Following observational studies, the presence of intraventricular dyssynchrony greatly improved the percentage of responders. $^{7-10}$ Thereby Doppler echocardiographic imaging emerged as an important tool for evaluating patients before CRT and numerous cut-off values of different parameters obtained by several echocardiographic modalities have been published. However, the recent PROSPECT (Predictors of Response to CRT) trial, designed to define which echocardiographic parameters might reliably predict clinical benefits, was quite disappointing. No single echocardiographic criterion of dyssynchrony could be recommended for patient selection before CRT implantation. Efforts are thus required to reduce the variability arising from technical and interpretative factors and to improve their predictive value. ${ }^{11}$ Other echocardiographic modalities such as dobutamine stress echocardiography, exercise echocardiography and new echocardiographic technologies (LV deformation using the speckle tracking method and 3D echocardiography) may emerge as new tools for a more accurate prediction of response to CRT.

\section{INTEREST OF STRESS ECHOCARDIOGRAPHY}

Several clinical and echocardiographic parameters have been used to assess outcomes from CRT and to define responders. Common clinical end points include subjective measures applying NYHA classification or quality of life questionnaire scores, objective parameters (death from heart failure, hospitalisation for, or associated with, worsening heart failure, heart transplantation, $6 \mathrm{~min}$ walk distance) or clinical composite scores including both subjective and objective measures of clinical status. Long-term response is identified when the patients' clinical status improves, mostly at 6 months of CRT. Many clinical trials have also used echocardiographic rest parameters, mainly LVEF and LV end-systolic volume, to assess LV reverse remodelling. LV end-systolic volume has been shown to be an independent predictor of prognosis. ${ }^{12}$ Response to CRT is thus also considered if the LV end-systolic volume decreases by 


\section{Box 1 Cardiac resynchronisation therapy indications}

- Optimal New York Heart Association class III/IV, LVEF $<35 \%$ and QRS > $120 \mathrm{~ms}$ (class I, level A)

- To improve symptoms/reduce hospitalisation (class I, level A)

- To reduce mortality (class I, level A)

ESC guidelines for the diagnosis and treatment of acute and chronic heart failure.

$>15 \%$ at 6 months or if LVEF increases by $>5 \% .{ }^{13-15}$ As previously discussed, the response to CRT appears complex, involving different mechanisms. More patients exhibit improvement in clinical status than show improvement in echocardiographic parameters. Stress echocardiography may emerge as a useful tool for assessing the dynamic component of exercise-induced changes in LV dyssynchrony, mitral regurgitation (MR) and the presence and extent of LV regional and global contractile reserve.

\section{EXTENT OF LV MECHANICAL DYSSYNCHRONY}

Numerous studies have suggested that the presence and extent of LV dyssynchrony, characterising the mechanical intraventricular dyssynchrony, might be a better predictor of response to CRT than ORS duration. ${ }^{7-10}$ Different Doppler echocardiographic techniques have been used to quantify LV dyssynchrony: M-mode, pulsed-wave tissue Doppler imaging (TDI), colour-coded TDI, tissue synchronisation imaging, strain by the speckle tracking method. ${ }^{16}$

However, LV dyssynchrony at rest is possibly not sufficient. An argument comes from the occurrence of important individual changes of LV synchronicity between rest and exercise, in the absence of inducible ischaemia. Lancellotti et al were the first to demonstrate the dynamic characteristic of LV dyssynchrony. ${ }^{17}$ The study showed important changes in LV synchronicity during exercise in most patients, with these dynamic changes varying substantially from patient to patient. Exercise-induced changes were adverse-increased LV dyssynchrony-in $46 \%$ of patients, whereas exercise improved the synchronicity of the LV in $43 \%$ (figure 1). No change between rest and exercise was identified in $11 \%$ of patients. Lafitte et al also applied exercise echocardiography to refine this potential exercise-induced asynchronous activation of the basal LV segments. ${ }^{18}$ Patients presenting exercise-induced LV dyssynchrony had poor exercise capacity, owing to decreased LV stroke volume and increased MR. Patients with this type of dyssynchrony may benefit from CRT and improve their exercise capacity, whereas patients who 'resynchronise' during exercise possibly might not benefit from CRT. Rocchi et al have evaluated the impact of exercise on electromechanical activation of the LV basal segments and found that exercise may induce a spatial change of LV dyssynchrony. ${ }^{19}$ In addition, they identified the value of exercise LV dyssynchrony for predicting functional improvement and LV reverse remodelling after CRT. ${ }^{19}$

Recently, low-dose dobutamine stress echocardiography was also proposed before CRT. ${ }^{20}$ During the isovolumic contraction, a characteristic abnormal septal motion is detectable on standard M-mode in the presence of left bundle branch block or right ventricular pacing. The authors hypothesised that these early and short-lived septal motions, the so-called septal flash, might be used as a marker of left bundle branch block-induced dyssynchrony. They demonstrated that dobutamine increased or unmasked septal flash. All patients were responders and had a resolution of septal flash after CRT.

\section{SEVERITY OF MR}

Patients with LV dysfunction frequently have functional MR (figure 2). There is an imbalance between LV closing and tethering forces, leading to mitral valve geometry deformation. ${ }^{21}$ Therapeutic interventions that aim at improving LV efficiency should conceptually reduce the severity of functional MR. It has been suggested that the response to CRT might be modulated by the presence of functional MR before implantation. The CAREHF study, including 813 patients, demonstrated that, as compared with responders, patients who had no significant MR were not improved by CRT. ${ }^{4}$ Indeed, it appears that CRT acutely reduces functional resting MR by an increase in $\mathrm{LV}$ closing force mediated by a rise in LV systolic pressure owing to more coordinated LV contraction and a more coordinated timing of mechanical activation of papillary muscle insertion sites. ${ }^{22} 23$ This benefit remains dependent on persistent pacing. ${ }^{24}$

Recently, Maréchaux et al suggested that the presence of functional MR at rest might be of importance for predicting acute haemodynamic response to CRT during exercise. ${ }^{25}$ They demonstrated that an acute positive response to CRT appeared only in
Figure 1 Rest transthoracic echocardiography demonstrating significant delay between anterior and inferior and posterolateral and anteroseptal walls by colour tissue Doppler imaging. In this patient, exercise induces a 'resynchronisation' of the left ventricular walls. Exer, exercise.

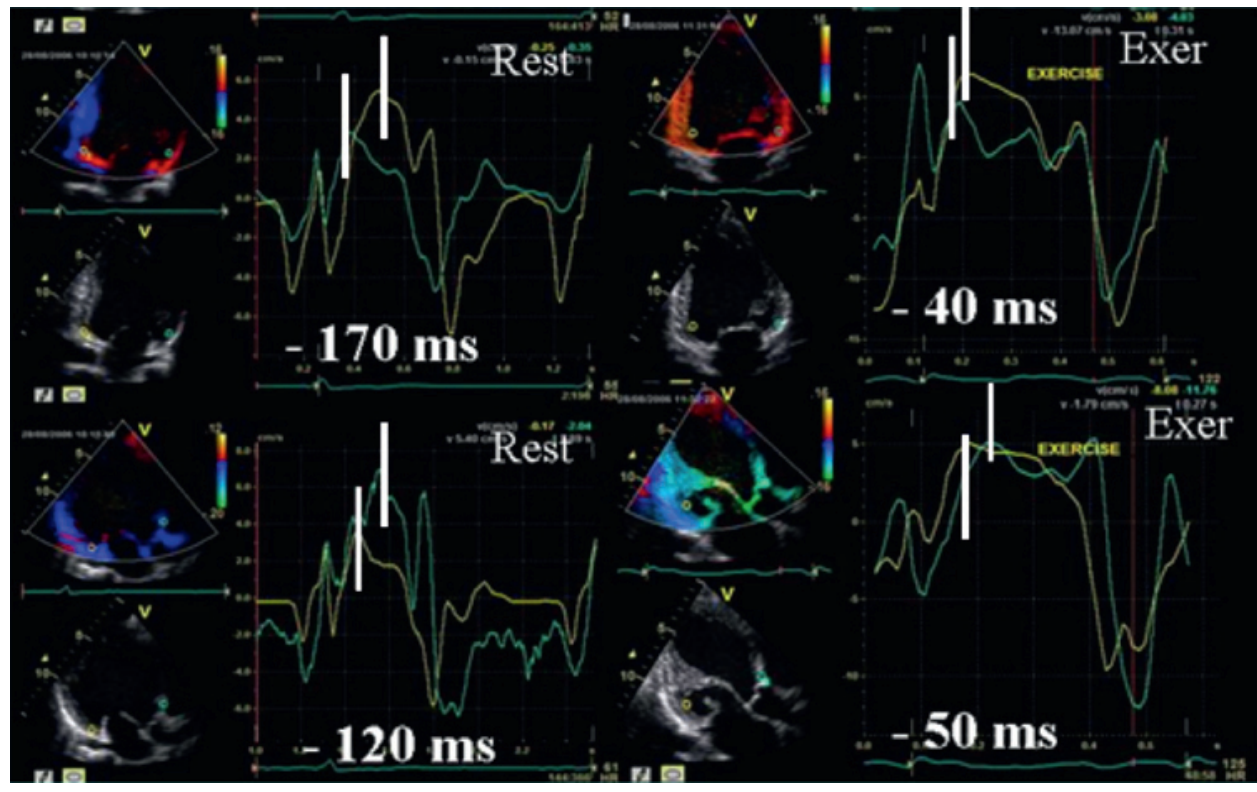


Figure 2 Left panel demonstrates mild functional mitral regurgitation in a patient with ischaemic cardiomyopathy at rest. Right panel shows an exercise increase in mitral regurgitation that becomes moderate. ERO, effective regurgitant orifice; MR, mitral regurgitation; PISA, proximal isovelocity surface area; $V$, velocity.
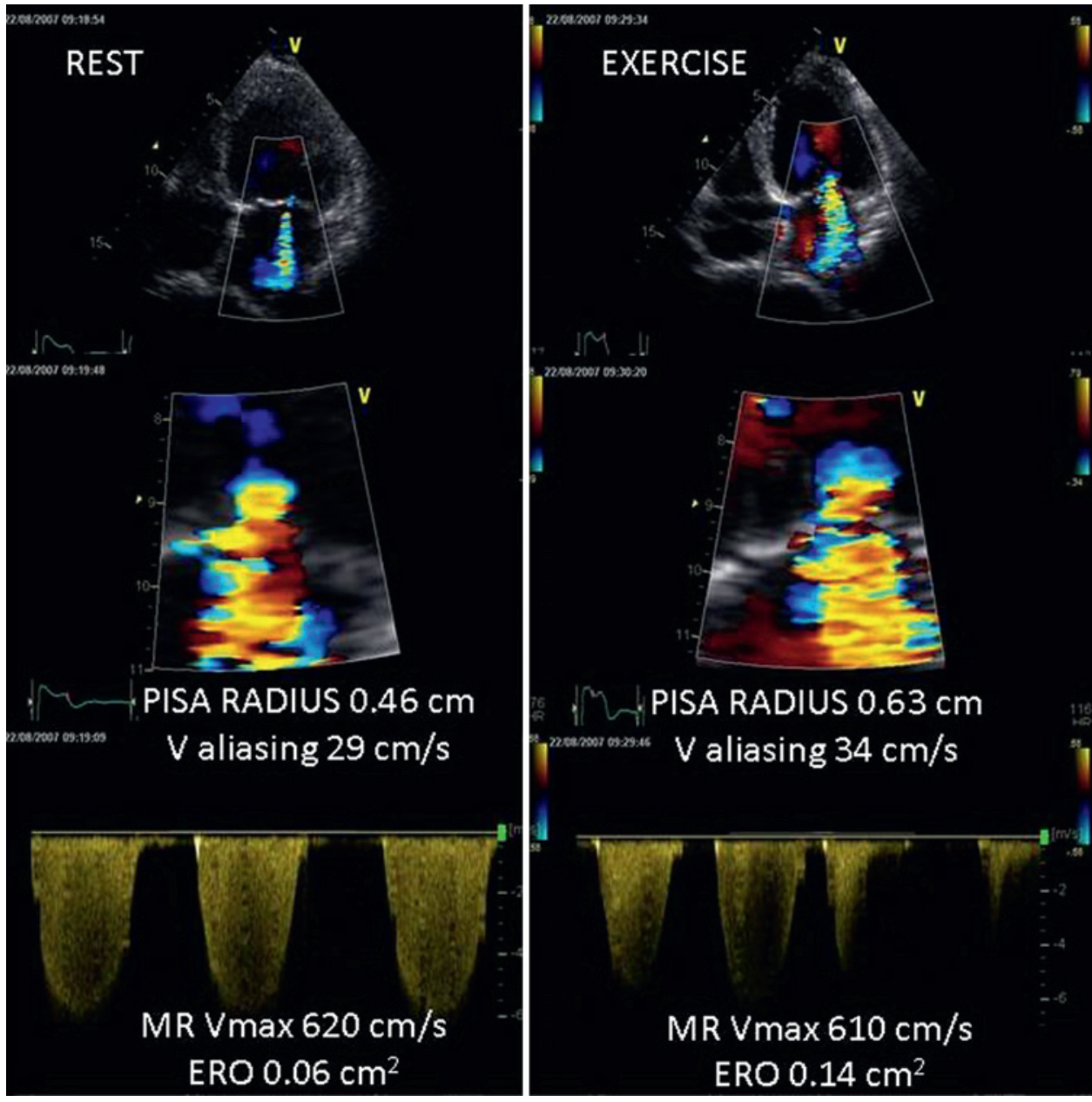

patients with functional MR at rest. Nonetheless, these results must be interpreted cautiously because none of the patients without $M R$ at rest developed $M R$ during exercise and also because LV dyssynchrony was not considered. Functional MR is frequently dynamic during exercise and is related to dynamic distortion of mitral valve geometry and to dynamic LV dyssynchrony. ${ }^{17}{ }^{26}$ CRT has also been shown to attenuate exerciseinduced increase in MR. ${ }^{27}$ The mechanism and the time course of exercise-induced decrease in MR after CRT have been described by Madaric et al. ${ }^{28}$ CRT is associated with a significant decrease in resting MR immediately after CRT, whereas exercise-induced MR remains stable. At 3 months, CRT-induced reverse LV remodelling and more favourable geometry of the mitral apparatus lead to a reduction of resting and also of exercise-induced $M R$, associated with improved cardiopulmonary performance during exercise. ${ }^{28}$

\section{ASSESSMENT OF CONTRACTILE RESERVE}

In chronic heart failure, the myocardium undergoes a remodelling process, including progressive fibrosis and loss of contractile and conduction properties. Another important factor that might explain the lack of efficacy of CRT is the presence of scar in the region of latest activation. Several trials have assessed the presence of myocardial viability before CRT implantation. Myocardial viability can be assessed globally or regionally, in the target region for the LV lead, and both appear to be important parameters to predict success of CRT.

The impact of scar tissue in the LV lead target site was first suggested from an anecdotal clinical report. ${ }^{29}$ Several clinical trials have investigated the importance of regional myocardial viability using different non-invasive imaging techniques. ${ }^{30} 31$ The absence or presence of scar tissue in the posterolateral wall, and its transmural extent, have been assessed using contrast-enhanced cardiovascular magnetic resonance. It has been demonstrated that only patients with severe baseline LV dyssynchrony and without transmural posterolateral scar have an excellent response rate (95\%). ${ }^{30}$ In contrast, all other patients - no dyssynchrony and/or posterolateral scar-exhibited a low response rate after CRT. Lim et al studied the importance of combined regional contractile reserve (CR) and LV dyssynchrony, 1 week after successful CRT implantation, in three conditions: (1) with the CRT device on; (2) 5 min after CRT device deactivation and (3) finally, during lowdose dobutamine infusion with the device on. ${ }^{31}$ In patients with CR in the LV lead target site (defined by segmental wall motion improvement by $\geq 1$ grade under dobutamine) LV dyssynchrony was significantly reduced with the CRT device on. The absence of CR was associated with a non-significant improvement of mechanical dyssynchrony. The presence of both CR and CRT induced a $22 \%$ increase of LV stroke volume in the presence of LV dyssynchrony, while no increase was observed in the absence of CR despite LV dyssynchrony.

In patients with depressed LV function, the identification of a global CR with stress echocardiography has been shown to provide important prognostic information. ${ }^{32}$ Recently, different clinical trials attempted to demonstrate that in patients referred for CRT, the presence of residual global myocardial viability might modulate the response to CRT. ${ }^{33-37}$ Assessment of global $\mathrm{CR}$ is accurate using contrast-enhanced cardiovascular magnetic resonance, SPECT, low-dose dobutamine echocardiography, 

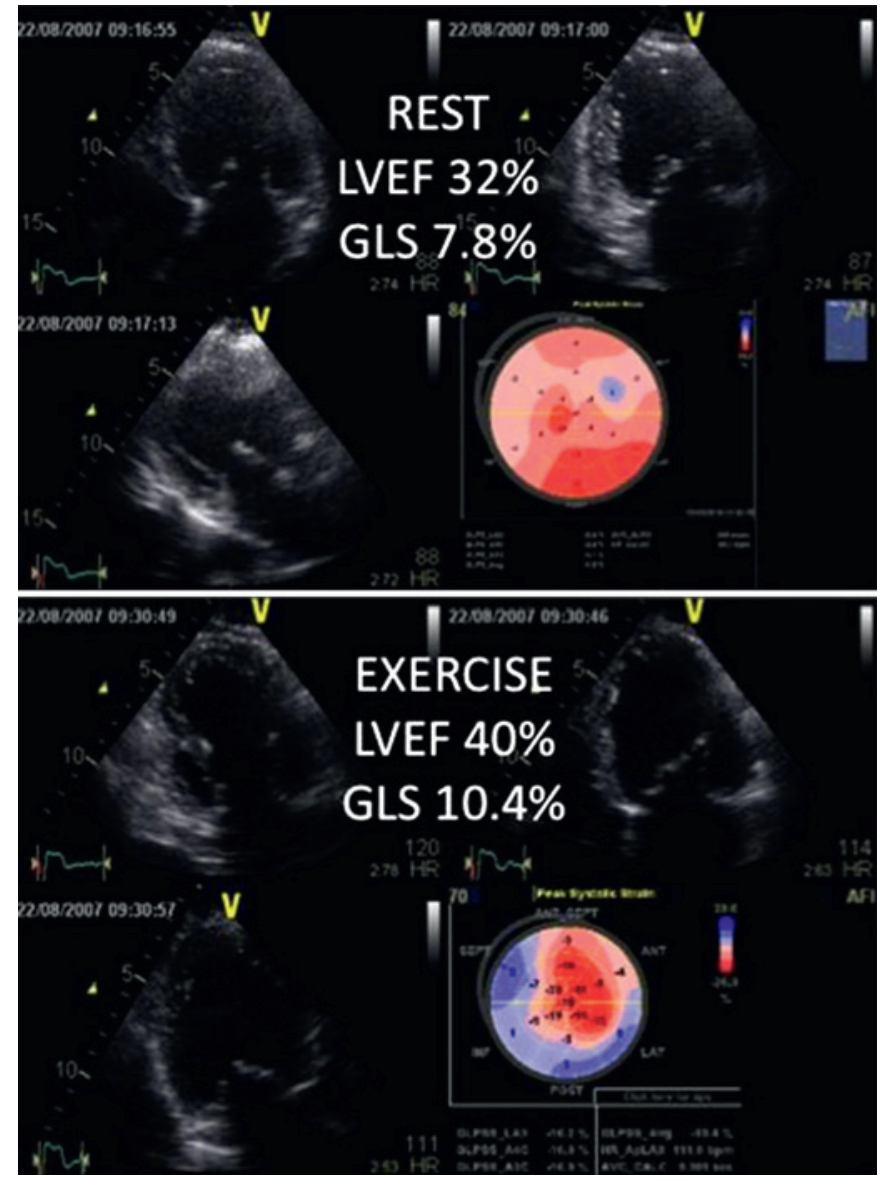

Figure 3 Upper panel shows reduced left ventricular ejection fraction $(32 \%)$ obtained by Simpson biplane method. Global longitudinal strain is reduced $(7.8 \%)$. Lower panel identifies the presence of global contractile reserve, demonstrated by an exercise increase of left ventricular ejection fraction by $8 \%$ and global longitudinal strain by $2.6 \%$. GLS, global longitudinal strain; LVEF, left ventricular ejection fraction.

myocardial contrast echocardiography or exercise echocardiography, and some of these techniques have already been applied to the assessment of global CR before CRT implantation. When low-dose dobutamine echocardiography is used, the presence of global CR appears to be an independent predictor of event-free survival after $\mathrm{CRT}^{34}{ }^{34}$ It has been recently shown that CR was present in $78 \%$ of clinical responders, whereas dyssynchrony was equally present in the responders and the non-responders. ${ }^{38}$ This finding was confirmed by Ypenburg et al, who showed that CR, defined as a $\geq 7.5 \%$ dobutamine-induced increase in LVEF, could predict reverse remodelling 6 months after CRT implantation. ${ }^{35}$

We recently focused our interest on the potential of exercise echocardiography to predict acute response to CRT (figure 3).

\section{Box 2 Interest of stress echocardiography: key points}

- Cardiac resynchronisation therapy (CRT) is recommended in selective patients with drug refractory systolic heart failure, but $30 \%$ of patients are non-responders

- No single rest echocardiographic criterion of dyssynchrony could be recommended for patient selection for CRT implantation. Other echocardiographic modalities such as dobutamine or exercise stress echocardiography may offer solutions

- Response to CRT appears complex, entangling different mechanisms

- Left ventricular dyssynchrony appears to have a dynamic behaviour that exercise testing can unmask

- Exercise-induced left ventricular dyssynchrony appears to be predictive of functional improvement and left ventricular remodelling after CRT

- CRT has been shown to attenuate mitral regurgitation increase during exercise

- Myocardial viability can be assessed globally or regionally, in the site of the left ventricular lead, using low-dose dobutamine infusion or exercise echocardiography

- Absence of regional contractile reserve is associated with non-significant improvement of mechanical dyssynchrony

- Global contractile reserve is an independent predictor of event-free survival after CRT

Baseline clinical and echocardiographic parameters of responders and non-responders were similar, except for a higher degree of $M R$, a greater extent of LV dyssynchrony and a higher myocardial deformation rate by $2 \mathrm{D}$ speckle tracking imaging in the $\mathrm{LV}$ lead region in responders. During exercise, an increase in LVEF by $\geq 6.7 \%$ and/or an increase in local strain by $\geq 2 \%$ were found to be predictive for response to CRT. Improvement in LVEF at peak exercise before multisite pacing correlated with improvement in forward LV stroke volume early after CRT. ${ }^{39}$ More recently, we have demonstrated that a $6.5 \%$ exercise-induced increase in LVEF yielded a sensitivity of $90 \%$ and a specificity of $85 \%$ to predict late response to $\mathrm{CRT}^{40}$ Rocchi et al also applied exercise echocardiography in this indication and determined that regional CR in the pacing site of the LV lead was associated with response to CRT at a 6 month follow-up. ${ }^{19}$

These concordant results using different techniques and different markers of myocardial viability highlight the potential interest in using CRT in less advanced stages of heart failure when reverse remodelling is still possible. ${ }^{35} 41$ Routine assessment of regional myocardial viability before CRT is of particular interest in patients with ischaemic cardiomyopathy. However, patients should not be refused CRT implantation only on the

Table 1 Exercise echocardiographic assessment before cardiac resynchronisation therapy implantation

\begin{tabular}{|c|c|c|c|}
\hline & Index & Setting & Method \\
\hline LV mechanical dyssynchrony & LV dyssynchrony, ms & $\begin{array}{l}\text { Colour TDI (frame rates } \geq 115 / s \text { ), apical } \\
2 \text { - and } 4 \text {-CV }\end{array}$ & $\begin{array}{l}\text { Time between peak of systolic velocity of the opposite } \\
\text { wall (septal-lateral, inferior-anterior, } \\
\text { posterolateral-anteroseptal), at rest and during } \\
\text { exercise }\end{array}$ \\
\hline Mitral regurgitation & $\mathrm{ERO}, \mathrm{mm}^{2}$ & $\begin{array}{l}\text { Colour Doppler (aliasing velocity }<10 \% \text { of } \\
\text { maximal velocity of the regurgitant jet), } \\
\text { apical } 4 \text {-CV }\end{array}$ & PISA method, at rest and during exercise \\
\hline Contractile reserve & $\begin{array}{l}\text { Global LV contractile reserve } \\
\text { Regional contractile reserve } \\
\text { (in the LV lead site) }\end{array}$ & $\begin{array}{l}2 D \text { gray scale images (frame rates } \geq 70 \text { / } \\
\text { s), apical } 2-, 3 \text { - and } 4-C V\end{array}$ & $\begin{array}{l}\text { LVEF (Simpson biplane), from rest to peak exercise } \\
\text { Speckle tracking analysis at the possible LV lead } \\
\text { site, from rest to peak exercise }\end{array}$ \\
\hline
\end{tabular}

CV, chamber view; ERO, effective regurgitant orifice; LV, left ventricular; LVEF, left ventricular ejection fraction; PISA, proximal isovelocity surface area; TDI, tissue Doppler imaging. 
basis of the absence of regional or global CR, since some patients felt they had improved despite the absence of viability. The results only underline the importance of an appropriate LV lead position. If the venous anatomy is not appropriate (LV lead position in front of transmural scar), a minimally invasive surgical approach should be performed.

\section{CONCLUSION}

The identification of potential responders to CRT before implantation can be improved by using different imaging modalities. Stress echocardiography appears to be an interesting tool (box 2). Resting and exercise LV dyssynchrony and functional MR severity assessments, as well as identification of the latest LV wall activated during exercise, appear to be useful before CRT implantation. Furthermore, global and regional CR, obtainable by dobutamine or exercise echo, seem to be reliable for predicting CRT response. Exercise echocardiographic parameters used in the assessment of patient before CRT are detailed in the table 1. The current findings shift the interest from electrical and mechanical dyssynchrony to the definition of myocardial substrate predicting response to CRT. Cardiovascular magnetic resonance is another possible alternative for identifying transmural scar and viable myocardium. Similar information can be obtained by multislice computed tomography with the advantage of visualising the patient's venous anatomy which helps in selecting the optimal lead position.

Competing interests None.

Provenance and peer review Not commissioned; externally peer reviewed.

\section{REFERENCES}

1. Vardas PE, Auricchio A, Blanc JJ, et al. European Society of Cardiology; European Heart Rhythm Association. Guidelines for cardiac pacing and cardiac resynchronization therapy: The Task Force for Cardiac Pacing and Cardiac Resynchronization Therapy of the European Society of Cardiology. Developed in collaboration with the European Heart Rhythm Association. Eur Heart J 2007;28:2256-95.

2. Dickstein K, Cohen-Solal A, Filippatos G, et al. ESC Guidelines for the diagnosis and treatment of acute and chronic heart failure 2008: the Task Force for the Diagnosis and Treatment of Acute and Chronic Heart Failure 2008 of the European Society of Cardiology. Developed in collaboration with the Heart Failure Association of the ESC (HFA) and endorsed by the European Society of Intensive Care Medicine (ESICM). Eur Heart J 2008:29:2388-442.

3. Bristow MR, Saxon LA, Boehmer J, et al. Comparison of Medical Therapy, Pacing, and Defibrillation in Heart Failure (COMPANION) Investigators. Cardiacresynchronization therapy with or without an implantable defibrillator in advanced chronic heart failure. N Engl J Med 2004;350:2140-50.

4. Cleland JG, Daubert JC, Erdmann E, et al. Cardiac Resynchronization-Heart Failure (CARE-HF) Study Investigators. The effect of cardiac resynchronization on morbidity and mortality in heart failure. N Engl J Med 2005;352:1539-49.

5. Leclercq C, Kass DA. Retiming the failing heart: principles and current clinical status of cardiac resynchronization. J Am Coll Cardiol 2002;39:194-201.

6. Hawkins NM, Petrie MC, MacDonald MR, et al. Selecting patients for cardiac resynchronization therapy: electrical or mechanical dyssynchrony? Eur Heart $J$ 2006;27:1270-81.

7. Yu CM, Gorcsan J 3rd, Bleeker GB, et al. Usefulness of tissue Doppler velocity and strain dyssynchrony for predicting left ventricular reverse remodeling response after cardiac resynchronization therapy. Am J Cardiol 2007;100:1263-70.

8. Bax JJ, Bleeker GB, Marwick TH, et al. Left ventricular dyssynchrony predicts response and prognosis after cardiac resynchronization therapy. J Am Coll Cardiol 2004:44:1834-40

9. Pitzalis MV, lacoviello M, Romito R, et al. Ventricular asynchrony predicts a better outcome in patients with chronic heart failure receiving cardiac resynchronization therapy. J Am Coll Cardiol 2005;45:65-9.

10. Notabartolo D, Merlino JD, Smith AL, et al. Usefulness of the peak velocity difference by tissue Doppler imaging technique as an effective predictor of response to cardiac resynchronization therapy. Am J Cardiol 2004:94:817-20.

11. Chung ES, Leon AR, Tavazzi L, et al. Results of the predictors of response to CRT (PROSPECT) trial. Circulation 2008;117:2608-16.

12. Yu CM, Bleeker GB, Fung JW, et al. Left ventricular reverse remodeling but not clinical improvement predicts long-term survival after cardiac resynchronization therapy. Circulation 2005;112:1580-6.

13. Yu CM, Fung WH, Lin $\mathrm{H}$, et al. Predictors of left ventricular reverse remodelling after cardiac resynchronization therapy for heart failure secondary to idiopathic dilated or ischemic cardiomyopathy. Am J Cardiol 2003;91:684-8.
14. Yu CM, Abraham WT, Bax J, et al. Predictors of response to cardiac resynchronization therapy (PROSPECT) - study design. Am Heart J 2005;149:600-5

15. Yu CM, Fung JW, Zhang 0 , et al. Tissue Doppler imaging is superior to strain rate imaging and postsystolic shortening on the prediction of reverse remodeling in both ischemic and nonischemic heart failure after cardiac resynchronization therapy. Circulation 2004;110:66-73

16. Gorcsan J 3rd, Abraham T, Agler DA, et al. Echocardiography for cardiac resynchronization therapy: recommendations for performance and reporting-a repor from the American Society of Echocardiography Dyssynchrony Writing Group endorsed by the Heart Rhythm Society. J Am Soc Echocardiogr 2008;21:191-213.

17. Lancellotti $\mathbf{P}$, Stainier PY, Lebois $F$, et al. Effect of dynamic left ventricular dyssynchrony on dynamic mitral regurgitation in patients with heart failure due to coronary artery disease. Am J Cardiol 2005;96:1304-7.

18. Lafitte $\mathbf{S}$, Bordachar $\mathrm{P}$, Lafitte $\mathrm{M}$, et al. Dynamic ventricular dyssynchrony: an exercise-echocardiography study. J Am Coll Cardiol 2006;47:2253-9.

19. Rocchi G, Bertini M, Biffi M, et al. Exercise stress echocardiography is superior to rest echocardiography in predicting left ventricular reverse remodelling and functional improvement after cardiac resynchronization therapy. Eur Heart J 2009;30:89-97.

20. Parsai C, Baltabaeva A, Anderson L, et al. Low-dose dobutamine stress echo to quantify the degree of remodelling after cardiac resynchronization therapy. Eur Heart J 2009;30:950-8.

21. He S, Fontaine AA, Schwammenthal E, et al. Integrated mechanism for functional mitral regurgitation: leaflet restriction versus coapting force: in vitro studies. Circulation 1997:96:1826-34.

22. Breithardt $\mathbf{O A}$, Sinha AM, Schwammenthal E, et al. Acute effects of cardiac resynchronization therapy on functional mitral regurgitation in advanced systolic heart failure. J Am Coll Cardiol 2003;41:765-70.

23. Kanzaki H, Bazaz R, Schwartzman D, et al. A mechanism for immediate reduction in mitral regurgitation after cardiac resynchronization therapy: insights from mechanical activation strain mapping. J Am Coll Cardiol 2004;44:1619-25.

24. Brandt RR, Reiner C, Arnold R, et al. Contractile response and mitral regurgitation after temporary interruption of long-term cardiac resynchronization therapy. Eur Heart J 2006;27:187-92

25. Maréchaux S, Pinçon C, Gal B, et al. Functional mitral regurgitation at rest determines the acute hemodynamic response to cardiac resynchronization therapy during exercise: an acute exercise echocardiographic study. J Am Soc Echocardiogr 2009;22:464-71.

26. Lancellotti $\mathbf{P}$, Lebrun F, Piérard LA. Determinants of exercise-induced changes in mitral regurgitation in patients with coronary artery disease and left ventricular dysfunction. J Am Coll Cardiol 2003:42:1921-8.

27. Lancellotti $\mathbf{P}$, Mélon $P$, Sakalihasan N, et al. Effect of cardiac resynchronization therapy on functional mitral regurgitation in heart failure. Am J Cardiol 2004;94:1462-5.

28. Madaric J, Vanderheyden M, Van Laethem C, et al. Early and late effects of cardiac resynchronization therapy on exercise-induced mitral regurgitation: relationship with left ventricular dyssynchrony, remodelling and cardiopulmonary performance. Eur Heart J 2007:28:2134-41.

29. Kanhai SM, Viergever EP, Bax JJ. Cardiogenic shock shortly after initial success of cardiac resynchronization therapy. Eur J Heart Fail 2004;6:477-81.

30. Bleeker GB, Kaandorp TA, Lamb HJ, et al. Effect of posterolateral scar tissue on clinical and echocardiographic improvement after cardiac resynchronization therapy. Circulation 2006:113:969-76.

31. Lim P, Bars C, Mitchell-Heggs L, et al. Importance of contractile reserve for CRT. Europace 2007;:9:739-43.

32. Ramahi TM, Longo MD, Cadariu AR, et al. Dobutamine-induced augmentation of left ventricular ejection fraction predicts survival of heart failure patients with severe nonischaemic cardiomyopathy. Eur Heart J 2001:22:849-56.

33. Hummel JP, Lindner JR, Belcik JT, et al. Extent of myocardial viability predicts response to biventricular pacing in ischemic cardiomyopathy. Heart Rhythm 2005:2:1211-17

34. Da Costa A, Thévenin J, Roche F, et al. Prospective validation of stress echocardiography as an identifier of cardiac resynchronization therapy responders. Heart Rhythm 2006:3:406-13.

35. Ypenburg C, Sieders A, Bleeker GB, et al. Myocardial contractile reserve predicts improvement in left ventricular function after cardiac resynchronization therapy. Am Heart J 2007;154:1160-5.

36. Ypenburg C, Schalij MJ, Bleeker GB, et al. Extent of viability to predict response to cardiac resynchronization therapy in ischemic heart failure patients. J Nucl Med 2006; 47:1565-70.

37. Chalil S, Foley PW, Muyhaldeen SA, et al. Late gadolinium enhancementcardiovascular magnetic resonance as a predictor of response to cardiac resynchronization therapy in patients with ischaemic cardiomyopathy. Europace 2007:9:1031-7.

38. Ciampi $\mathbf{0}$, Pratali L, Citro $\mathrm{R}$, et al. Identification of responders to cardiac resynchronization therapy by contractile reserve during stress echocardiography. Eur $J$ Heart Fail 2009;11:489-96.

39. Moonen M, Senechal M, Cosyns B, et al. Impact of contractile reserve on acute response to cardiac resynchronization therapy. Cardiovasc Ultrasound 2008;6:65.

40. Lancellotti $\mathbf{P}$, Senechal M, Moonen M, et al. Myocardial contractile reserve during exercise predicts left ventricular reverse remodelling after cardiac resynchronization therapy. Eur J Echocardiogr 2009;10:663-8.

41. Moss AJ, Hall WJ, Cannom DS, et al; MADIT-CRT Trial Investigators. Cardiacresynchronization therapy for the prevention of heart-failure events. N Engl J Med 2009;361:1329-38 